\title{
Evaluation of Mental Calculation Educational System with Invisible Abacus
}

\author{
Keita Tokuda \\ Department of Pharmacy, the \\ University of Tokyo Hospital, \\ Faculty of Medicine, the \\ University of Tokyo \\ 7-3-1 Hongo, Bunkyo-ku \\ Tokyo, Japan \\ tokudakeita- \\ tky@umin.ac.jp
}

\author{
Keiko Iwasa \\ Digika Co. Ltd. \\ 2-11-14 Fujimi Chiyoda-ku \\ Tokyo, Japan \\ iwasa@digika.jp \\ Naoya Fujiwara \\ Graduate School of \\ Information Sciences, Tohoku \\ University \\ 6-3-09 Aoba, Aramaki-aza \\ Aoba-ku, Sendai, Japan \\ fujiwara@se.is.tohoku.ac.jp
}

\author{
Kazuki Yoshida \\ Digika Co. Ltd. \\ 2-11-14 Fujimi Chiyoda-ku \\ Tokyo, Japan \\ yoshida@digika.jp
}

\section{Akihito Sudo}

Faculty of Informatics,

Shizuoka University

3-5-1 Johoku, Naka-ku,

Hamamatsu-shi, Shizuoka, Japan

sudo@inf.shizuoka.ac.jp

\begin{abstract}
We evaluate an educational system, "SoroTouch" developed for the acquisition of abacus based mental calculation skills efficiently. SoroTouch is an iPad app with a unique interface called "Invisible mode", where the beads are not displayed, and the student is required to visualize and operate the beads mentally to solve the problem. Furthermore, SoroTouch helps to train fast accurate management of the beads, while providing students with features to facilitate continuous learning. The analysis of user learning data revealed that in 2017 and 2018, 54\% and 62\% of withdrawing students successfully acquired mental calculation abilities by the time of their withdrawal, respectively. Time spent on SoroTouch for skill acquisition was verified to fall between 7 and 26 months. This time period is defined from the student's start of learning, until the student's demonstration of mental calculation abilities.
\end{abstract}

Permission to make digital or hard copies of all or part of this work for personal or classroom use is granted without fee provided that copies are not made or distributed for profit or commercial advantage and that copies bear this notice and the full citation on the first page. Copyrights for components of this work owned by others than ACM must be honored. Abstracting with credit is permitted. To copy otherwise, or republish, to post on servers or to redistribute to lists, requires prior specific permission and/or a fee. Request permissions from Permissions@ acm.org.

ICIET 2020, March 28-30, 2020, Okayama, Japan

(C) 2020 Association for Computing Machinery.

ACM ISBN 978-1-4503-7705-8/20/03 . . \$15.00.

DOI: https://doi.org/10.1145/3395245.3396417

\section{CCS Concepts}

-Human-centered computing $\rightarrow$ Human computer interaction (HCI);

\section{Keywords}

architectures for educational technology system; evaluation methodologies; human-computer interface; distance education and telelearning; elementary education; STEM education;

\section{INTRODUCTION}

Proficiency in mental calculation signifies the acquisition of a fundamental skill, regarded as a part of the basics of primary education. The idea of understanding and acquiring number sense and its concept remains significant today, even after the invention of calculators and computers. These abilities indicate the basics of understanding the notion of numbers [1, 2, 3, 3, 5, 6]. In contrast to Arabic numeral notation, which uses abstract symbols, Japanese abacus, or "soroban" expresses numbers intuitively and physically. Japanese abacus is comprised of freely-moving beads that are attached to multiple vertical columns, which are separated into two of decks (upper and lower) by a horizontal beam [7]. The lower deck consists of 4 or 5 beads per column and the upper deck consists of 1 or 2 beads per column. Starting from the first column on the right, the beads have the least numerical value, with the lower deck beads representing numerical values 1 - 4 in vertical order, and the upper deck bead representing the numerical value of 5. This trend continues from the next column onwards, with the next column's lower deck beads representing multiples of 10 with an upper deck 


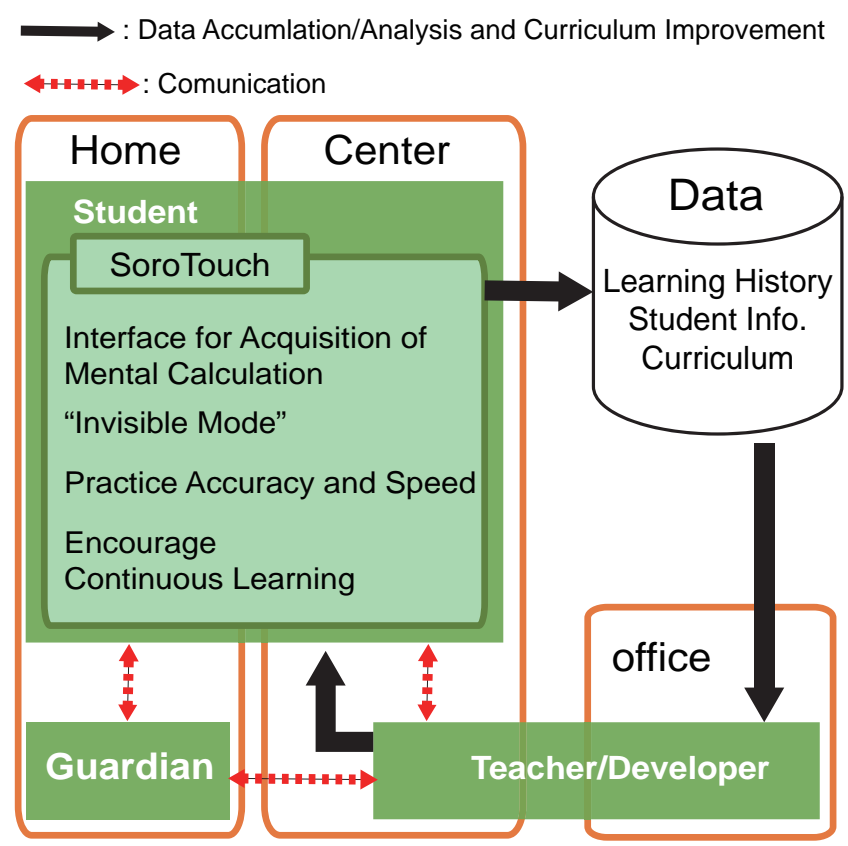

Figure 1: SoroTouch Systems, or the schematic diagram of obtained student learning data and the procedures of its implementations.

bead of 50 and the next column's lower deck beads representing multiples of 100 with an upper deck bead of 500, etc. The beads are considered to be "active" (in their numerical representation) when they are brought towards the beam. Therefore, the characteristic of expressing substantial numerical figures with a horizontal layout of beads functions within the base ten decimal system [7]. The unique structure of the abacus suggests its logical objective for young children to understand the notion of numbers, emphasizing the idea behind "digits." Indeed, the required elementary school curriculum recently developed by the Ministry of Education, Culture, Sports, Science, and Technology of Japan recommends students to learn how to express numbers and calculate elementary arithmetic using the abacus [8]. The capability of representing and understanding numbers through imaging the placement of beads potentially leads to students acquiring highly advanced calculation skills through this mental process [9, 10, 11, 12, 13, 14, 15.

The integration of information technology (IT) taking place in the educational field facilitates the analysis of the learning status, skills, data, and progress of individual students to further develop the educational system. Overcoming the challenges in the educational system was difficult until the recent incorporation of IT, which has been attracting global attention for solving the challenging elements listed below. The introduction of IT into education is expected to overcome the following difficulties: (1) interactive learning using user interface (UI) via touchscreen function, (2) a seamless learning environment utilizing cloud computing both at home and center, (3) accumulating and managing individual student learning data, (4) assessing each student's academic skills, (5) customizing curriculum for individual students allowing adaptive learning, (6) analyzing big data generated by the learning process, (7) improving interregional educational inequality, (8) distributing educational contents to a large population, and (9) lower educational costs.Until now, the mechanism of learning the abacus did not involve much IT. However, introducing IT in learning abacus enables alterations of the interface flexible and eases the accumulation of learning historical data. Successful educational development is realized if managing each individual's manipulation process and learning progress can be done easily with low economic and labor costs. Based on the understanding of the abacus mastering process, this advancement improves effectiveness, as the curriculum can be customized to optimize the learning material for each student.

This report accounts an exemplified case of Digika Co. Ltd. who implements the learning of the abacus on iPads with their system, "SoroTouch," reflecting the purpose of what is mentioned above. Furthermore, the examination will include the system's detailed characteristics, incorporating a tablet device to learn mental calculation, and its quantitative assessment through analyzing their accumulating learning history data. While the iPad app, SoroTouch, relies on an abacus-like user interface (UI), its design heavily focuses on the efficient acquisition of mental calculation skill, out of all the different utilities of abacus calculation. Two features make up SoroTouch: "Visible Mode," which specializes on training finger movements to control the beads, and the patented technology, "Invisible Mode," which sharpens the learner's memory, as he or she must memorize the placement of the beads. The function of the "Invisible Mode" is what makes SoroTouch unique, and it is impossible to establish with the standard abacus. Additionally, through the scheme of gradually increasing the difficulty as the students study every day and complete stages, the application aims to provide fun for students while they learn. Managing operation on the cloud-computing server enables the use and application of each student's learning-record data.

The Method section of this report will introduce the specific methodology demonstrated by SoroTouch with their system of continuous studying and gaining of mental calculation skills in a short time period. In the Evaluation of SoroTouch section, the analysis will examine whether their continuous short term studying system works for practical use through the accumulated learning data of their students. Lastly, section 4 is the conclusion of the entire report.

\section{METHODS}

\subsection{The Characteristics of SoroTouch}

Digika Co. Ltd. has developed an original iPad app, SoroTouch [16], which trains its users to acquire the abacus method of mental calculation using their patented technology, Invisible Mod 1 Through the training for mental calculation, one must achieve three crucial steps in order to acquire the skill. The requirements include: quickly manipulating the beads in a way that accurately corresponds to the calculation; speedily and precisely imaging the formation of the beads according to the calculation rule; and continuing to practice consistently at a certain pace. The development of SoroTouch provided the resolution to these key ideas combining Visible Mode, which trains the operational aspect, and Invisible Mode designated for training and improving students' imaging skills. "Invisible mode" is the most important and unique mechanism in the SoroTouch interface. It is used for the direct acquisition of the abacus method of mental calculation. Since "Invisible Mode" does not display the beads, the student is required to visualize and operate the beads mentally to solve the problem. This property has been introduced in SoroTouch based on the idea that demanding the students to image mentally the beads, like abacus experts who have acquired an advanced mental calculation [9, 10, 11, 12, 13, 14, 15]), should facilitate the acquisition of mental calculation. In contrast, beads are always visualized with the usual abacus learning method.

${ }^{1}$ The video explaining "invisible mode" is available at https://youtu. be/BdWJb80nD-w 

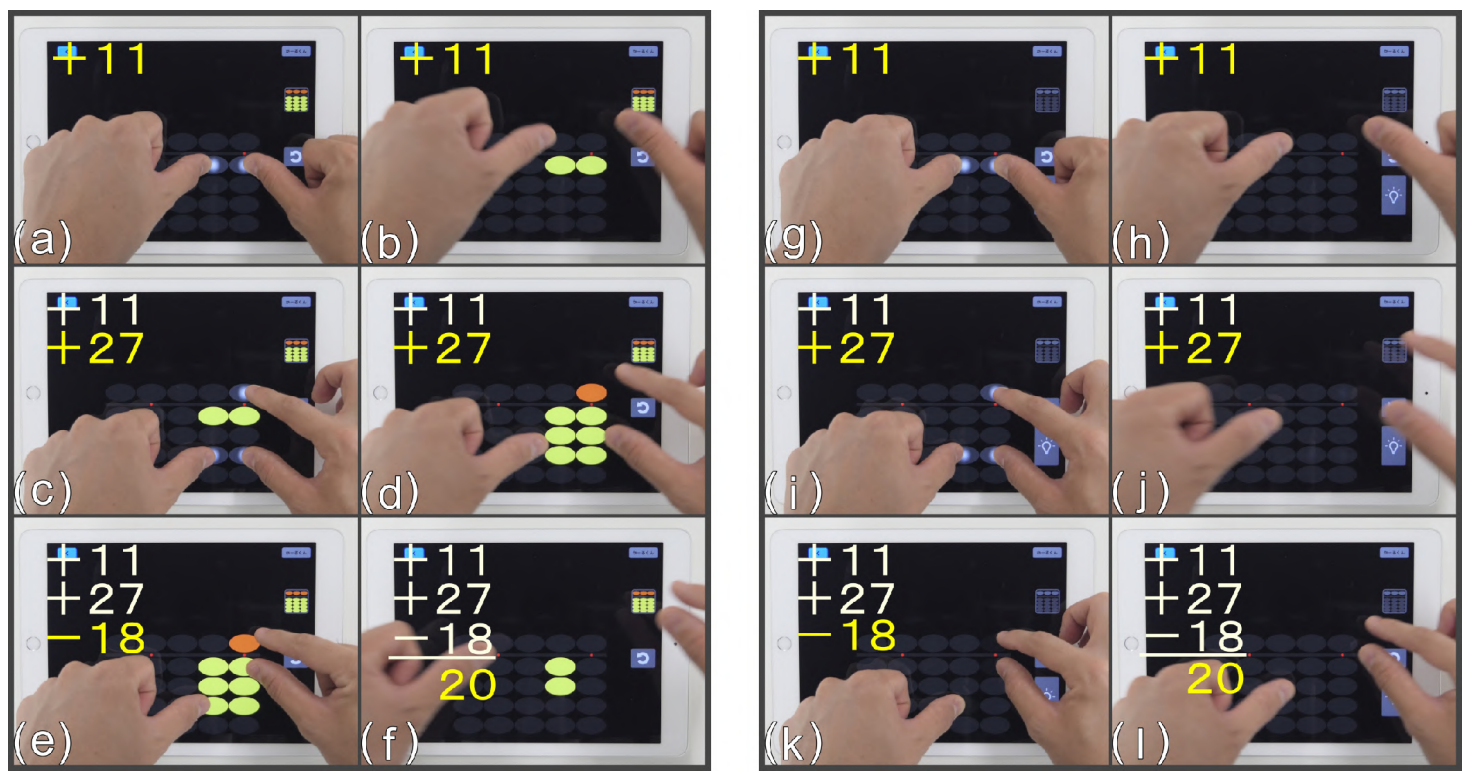

Figure 2: UI of SoroTouch, and procedural demonstration. (a)-(f) displays Visible Mode and (g)-(l) displays Invisible Mode. The counterparts demonstrate the same sample calculation, $11+27-18=20$. The decimal number represented in each column depicts the orange bead as a value of "five" and the yellow bead as a value of "one." The lighting beads total up to express a certain number value. By touching the screen, the beads light up which adds value accordingly. In the case of (a), the thumbs are touching "11", and (b) shows the touched beads lighting up as the fingers are released. Consequently, (c) demonstrates "27" added on to the initial value of "11" as the beads appears lit up in (d), corresponding to the value of " 38 " once the fingers are released. In contrast, touching beads that are already lit up will allow subtracting values, as "18" is deducted in (e). The results that the left over lit-up beads resembled in the case of (f) is "20," as shown after the fingers are released. The process of Invisible Mode demonstrated in (g)-(l) does not visibly show the beads, however, it corresponds to the procedures captured in (a)-(f).

Therefore, the abacus itself is not an active mechanism to acquire mental calculation. This difference makes SoroTouch unique.

Additionally, the app incorporated a further mechanism: designing an easier access for users to continue learning in a consistent manner. Figure 1 displays the educational system used by SoroTouch as well as the uses and applications of its obtained data. Other than the fact that it allows their students to work freely on the inapp installed math problems, SoroTouch also enables their students' teachers and guardians to use their curriculum at home, class, or office, for efficient skill acquisition. Furthermore, collecting all of their students' learning history data leads to the enhancement of their program for further development.

\subsection{The user interface of SoroTouch}

Figure 2 represents the user interface (UI) of SoroTouch implemented in the iPad systems. The beads are arranged similarly to a commonly used Japanese abacus. In other words, each column of five vertical beads represents one digits, marking the decimal number. The orange bead located on the very top of the column, indicates a value of five, while each of the yellow beads positioned below, symbolizes the value one. The total of displayed beads per column expresses the numbers of 0 to 9 . These implied rules replace the procedures of the four basic arithmetic operations. The placement of abacus beads expresses the decimal numbers, place values with the placement of its beads, and calculation is proceeded by positioning the beads up and down. SoroTouch then carries out calculation by tapping on the touch screen. In Visible Mode, the calculation is done by turning the lights of the beads on and off (Figure 2(a)-(f)). The calculation problems are provided in two separate ways: Reading allows for visually looking at the given problem off the screen, while listening dictates the given problem aimed for auditory comprehension. For reference, "SoroTouch Interface" [17] depicts an example working on the calculations, "Listening Practice" [18] demonstrates how the listening problems are done.

\section{3 "Visible mode"}

Students initially master the operational aspect of SoroTouch by practicing calculations using "Visible Mode." In this mode, the light of beads turns on and off by touching the screen with the fingers, which expresses the decimal number. The beads are represented in yellow and orange colors with an elliptical shape for the intention of having a residual image effect. From an auditory countermeasure, the pitch of the sound varies depending on the position of the beads as well as whether the beads are turning on and off. For instance, Figure 2(a) displays number "11" with "1" in both the tens and ones places. The calculation example of adding " 27 " to " 11 " then subtracting " 18 " is illustrated by displaying " 2 " in the tens place and " 7 " in the ones place in a supplementary manner (Figure 2(c)). This practice of touching the screen utilizing both hands results with the screen revealing the number "38" (Figure 2 (d)). As Figure 2 (e) exemplifies, extinguishing "1" from the tens place and "8" from the ones place enables subtraction. As a result, Figure 2(f) resembles the derived outcome of $11+27-18=20$. With the visible movements of the beads within this mode, students are expected to focus on gaining accuracy and speed.

\section{4 "Invisible mode"}

After getting used to the process in "Visible Mode," students proceed to conduct calculation in "Invisible Mode" (Figure 2 (g)-(l)). In this mode, the beads do not change colors but highlight in a dim 
white color for a short moment the instant the fingers and screen come into contact, allowing the confirmation of which beads were touched. Therefore, it requires students to touch the screen while imaging the arrangements and operations of the beads in the mind. Although the calculation process in examples, Figures 2 (g)-(1) and (a)-(f), are the same, calculating in "Invisible Mode" demands mentally imaging the positioning of abacus beads while conducting the procedures. This crucial skill is inevitably necessary for carrying out abacus method mental calculation. For the reason being, "Invisible Mode" possesses higher difficulty compared to "Visible Mode." "Invisible Mode" puts the manipulation of picturing the shape of beads in the head, as in abacus method mental calculation, into practice on to the iPad screen.

\subsection{The curriculum of SoroTouch}

The essential step in acquiring the mental calculation based on SoroTouch involves memorizing various procedures of the four arithmetic operations to conduct calculations in arbitrary situations. Digika Co. Ltd. analyzes these compulsory functions into practical elements and consolidates a curriculum designed to acquire the mental calculation based on SoroTouch. Specifically, the output factors include from basic arithmetic, like $1+1$ and single-digit multiplication, to the ones with greater difficulty level, like operations that involve regrouping, calculations with more digits, and addition of more numbers. Proceeding this curriculum proposes to naturally cultivate mental calculation abilities while instilling the necessary technical skills for the specialized calculation. Several math problems deal with each component consisting SoroTouch as a single unit, providing both "Reading" and "Listening" questions. Students are expected to acquire every element gradually one by one, starting from the basics. At first, all math problems given at every level are done in "Visible Mode," allowing visible calculations using beads that light on and off. After getting used to doing calculations in "Visible Mode," the app provides students with problems to solve using "Invisible Mode." In this manner, SoroTouch develops a curriculum that enhances operational accuracy and speed as it leads to practicing calculation without the beads visually displayed. Thereafter, the same practice is repeated for advanced levels, later in the curriculum.

\subsection{Accumulating learning history data, man- aging and applications}

The fact that SoroTouch is implemented in iPads makes it easy to obtain students' learning history data. As mentioned in Introduction, the characteristic of educational devices allows for the analysis of course materials with the collected data. Moreover, the informational technology constructs an adaptive learning environment where every student gets to work at his or her own pace. In the case of SoroTouch, the system accumulates and records, e.g. the number of questions solved, the time used to answer the questions, and the number of correct and incorrect answers. Furthermore, the app also records detailed data of where the fingers were wrongly placed to assist in improving mental calculation skills (Figure 1). SoroTouch enables each student to access their personal data from the display screen to verify what they are having difficulties with. Acknowledging the incorrect answers and their point of occurrence helps teachers to further understand the individual's accuracy of finger movements, speed, and the retention of imaging skills of the beads. One of the functions of SoroTouch permits the selection of these factors one wishes to improve so that the student can practice on a specific segment repeatedly. The students' learning data is also utilized to review the existing curriculum. For example, if numerous students were experiencing difficulties at a certain point, the ques-

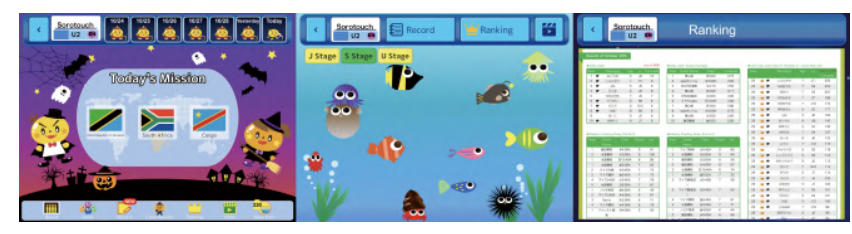

Figure 3: Elements to increase motivation. (a) Collecting flags and (b) Aqua Park. These components can be obtained by completing missions. (c) Verify student rankings directly from the app.

tion given in that point will be revised to an easier question in order to continuously improve the curriculum.

\subsection{Systems to keep up the motivation for learn- ing}

The structure of SoroTouch aims for students to enjoy learning with its various features (Figure 3). Students answer the questions contained in the curriculum to complete each mission and proceed to the next level; this is intended to provide the fun aspect of playing games, while acquiring skills for mental calculation (Figure 3 (a)). The system is designed such that one mission is completed per day on average and, once students reach a certain stage, they can collect incentives like flags and characters to add to their collection (Figure 3 (b)). Additionally, the reviews for areas of weakness also have a gamified structure. The comprehensive ranking lists (Figure 3 (c)) enables students and their parents/guardians to verify the student's progress. Students' learning data is also used in class to elevate their motivation. SoroTouch facilitates their class using their centerexclusive app, which allows the students' to work cooperatively in a gamified manner. Although the students can present what they have learned in front of other classmates, the opportunity will not effectively encourage them to learn if all students need to work on the same problems. Especially those who are progressing at a slower pace, this will not only enable them to solve the problems along with others but will also decrease their motivation to learn. The SoroTouch system however, grasps every students' progress. Thus, it customizes the essential set of problems for each student individually while working together as a class.

\subsection{Summary}

In summary, this section explored the characteristics of SoroTouch. The aim of easing the effort needed to acquire mental calculation abilities led to the development of this app. SoroTouch implements an active learning style IT system that enables their students to learn independently at home. The app has executed the following factors: Besides the "Visible Mode," founded on the original abacus, the introduction of the "Invisible Mode" effectively requires students to calculate using their minds. With thorough analysis of their students' data, the app automatically selects problems from the course that customarily suits each specific student. Moreover, understanding where the students are having trouble enables the teachers to communicate better with their parents/guardians. Additionally, displaying learning progress allows one to visually verify his or her improvement. All of the collected data is used to review and improve the curriculum.

\section{EVALUATION OF SOROTOUCH}

In order to evaluate the effectiveness of SoroTouch for mastering the mental calculation, we investigated the dataset accumulated from students who were enrolled in the SoroTouch curriculum. First, we 


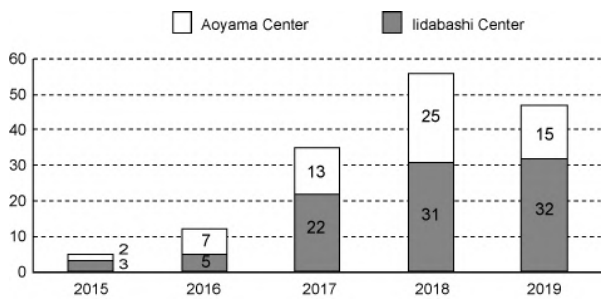

Figure 4: Number of students who completed or withdrew from the SoroTouch curriculum by year.

show the number and the ratio of students who were actually able to master mental calculation skills ${ }^{2}$ The following analyzed the period engaged with the SoroTouch curriculum to master mental calculation. Lastly, the average time and days spent on learning is evaluated.

There are two types of dataset necessary for the analysis reported in this section. First, we utilized the data on the mental calculation skill level of the students who completed or withdrew from the SoroTouch curriculum in order to reveal what proportion of the learners actually master the metal calculation and the amount of time needed for development of the skill. In addition, we accumulated the data on the learning status of all enrolled students to observe the time spent for studying the mental calculation. Both of the data in this report captures the students, who enrolled the SoroTouch curriculum between the age of 4 to 8 years at either SoroTouch Iidabashi Lab Center or SoroTouch Aoyama Center.

\subsection{Achievement Rate of Mastering Mental Cal- culation}

\subsubsection{Dataset}

In order to evaluate the effectiveness of SoroTouch for mastering mental calculation, we investigated the data of the students who were admitted to the SoroTouch curriculum after April 1, 2014 and those who quit in between the period of April 1, 2014 to December 31, 2018. April 1, 2014 is the day when Digika Co. Ltd started to specialize in mental calculation training using the SoroTouch app. The data includes a yearly breakdown of whether or not the students actually acquired mental calculation skills by the time of withdrawal from the SoroTouch curriculum, and the monthly statistics of the time it took for the students in the withdrawing group who acquired the skill. As shown in Figure 4 the yearly number of students who quit from year 2014 to 2018 is $5,12,35,56$, and 47 respectively. None of the students were age nine or older due to the specific enrollment policy of requiring only children aged eight or under.

The presented study excludes the data of students withdrawing due to family circumstances, such as relocating to a new residence or the need for caregiving, changing center location, or switching to the remote learning course. Students who appeared to withdraw within two months of enrollment were also excluded as withdrawing immediately following their admission.

\subsubsection{Result of Achievement Rate}

Figure 5 shows the achievement rate of mastering mental calculation. In the very first year the SoroTouch curriculum began in 2014, all five of their students were unable to master the mental calculation. In the following year however, two out of twelve students mastered the mental calculation by the time of completion

\footnotetext{
2"mastering mental calculation," as mentioned in this report, refers to acquiring the calculation ability described in Appendix.
}

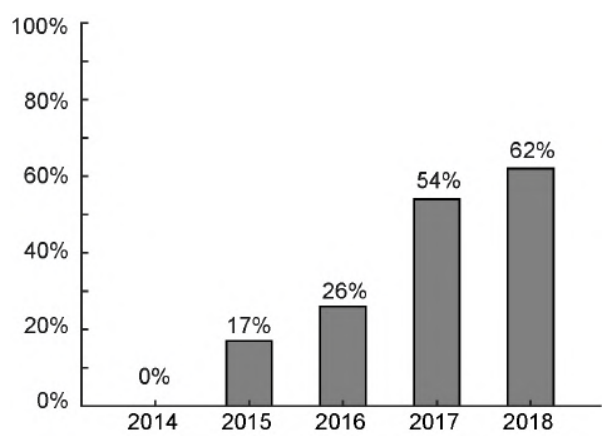

Figure 5: Proportion of students that mastered mental calculation by the time of completion or withdrawal of the SoroTouch curriculum.

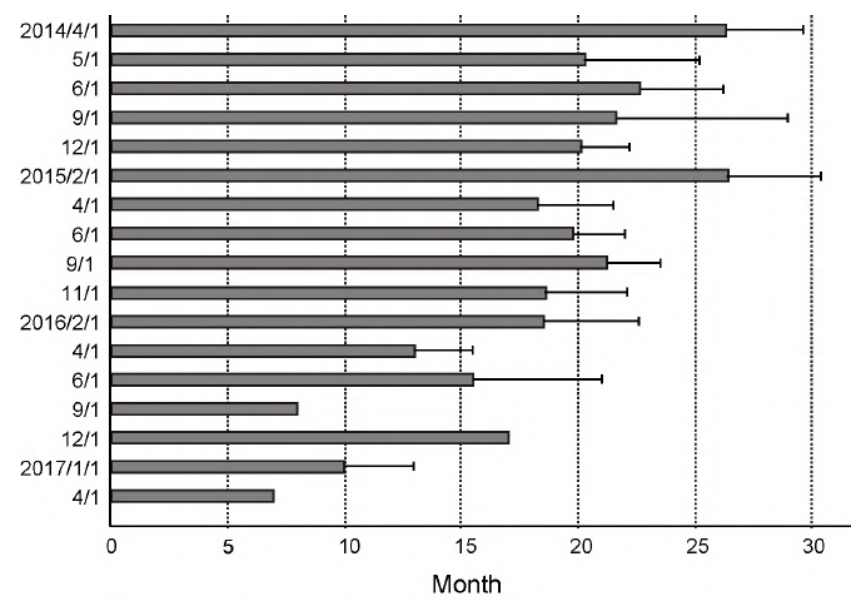

Figure 6: The time required to master the mental calculation by enrollment month.

of or withdrawal from the curriculum. After 2015, the number of students who mastered mental calculation by the time of completion of or withdrawal from the SoroTouch curriculum increased yearly: 9 students consisting 26\% in 2016, 30 students consisting 54\% in 2017 , and 29 students consisting $62 \%$ in 2018 . The extended learning time of each individual and curriculum improvements are the factors that potentially contributed to the increase in the acquisition rate. This notwithstanding, there are not many changes observed in terms of age and residential location within the selected student segment. Therefore, the natural qualities of the students and their initial knowledge at the time of enrollment do not play a role in the acquisition rate.

This result implies that approximately $60 \%$ of children, aged five to eight, who enrolled in the SoroTouch curriculum at Iidabashi Center or Aoyama Center can achieve a mastery of the mental calculation.

\subsection{Required Period for Mastering the Men- tal Calculation}

In this section, we analyze the data to show the time required for mastering the mental calculation using the same dataset described in the previous section. Selecting only the students who mastered the mental calculation out of the data, the length of time, measured from their time of enrollment to the time they mastered the mental calculation, reveals the average time required for skill development. Note 


\begin{tabular}{|c|c|c|}
\hline & \#students & Month \\
\hline 4 & 1 & 13.0 \\
\hline 5 & 26 & 22.1 \\
\hline 6 & 23 & 20.4 \\
\hline 7 & 17 & 16.9 \\
\hline 8 & 3 & 15.7 \\
\hline \hline Sum & 70 & 19.9 \\
\hline
\end{tabular}

Figure 7: Average required time to master the mental calculation by age.

that, the period explained above exemplifies the time of admission to the time they either passed 3rd Class Global Mental Calculation Test or completed all 720 SoroTouch Missions (see Appendix for details), emphasizing that the period was not until the time of their completion or withdrawal.

Figure 6 and 7 shows the time period needed for mastering the mental calculation in order of the month of enrollment. For the months not listed, like July and August 2014, the records indicate that either there were no enrolled students or none of the students enrolled mastered the mental calculation. In April 2014, averaging a period of 26 months was taken for the acquiring mental calculation abilities. The average required period to master the skill gradually shortens as follows: February 2015 was about 26 months, June 2015 was about 20 months, April 2016 was about 13 months, June 2016 was about 15 months, and lastly April 2017 was about 7 months.

This result suggests that spending 7 to 26 months studying the mental calculation with the SoroTouch curriculum will lead to mastering the mental calculation. The factors influencing the decrease of the period required for developing the skill is potentially due to the constant improvements of SoroTouch, as exemplified by its curriculum and learning systems. This notwithstanding, there are not many changes observed in terms of age and residential location within the selected student segment. Therefore, the natural qualities of the students and their initial knowledge at the time of enrollment do not to play a role in the growth rate.

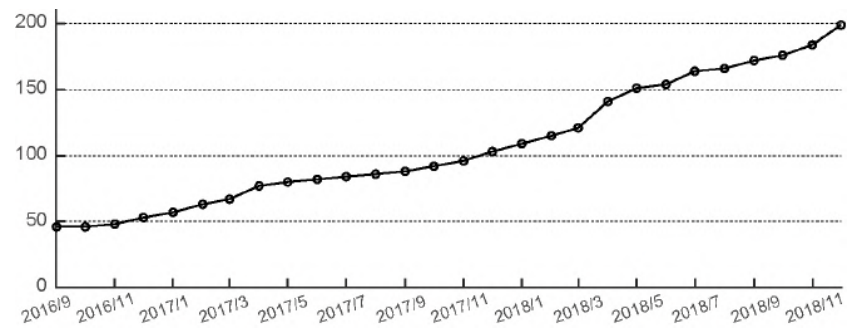

Figure 8: The number of registered students subjected for data analysis.

\subsection{Time Spent for the SoroTouch Curricu- lum}

\subsubsection{Dataset}

In order to confirm that the time spent on learning with SoroTouch falls within the average range of time used for other early childhood extracurricular activities or lessons, this section considers the length of time spent per day and the number of days spent per month precisely on SoroTouch. We analyzed the learning history data of all 199 students who were enrolled at the time of Dec. 31, 2018. Figure 8 illustrates a shift in the number of registered students subjected to data analysis. The male to female ratio, $51 \%$ to $49 \%$, (a)

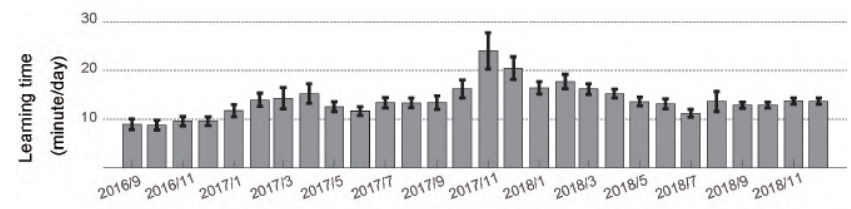

(b)

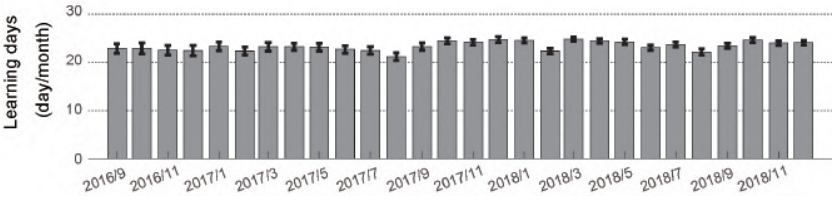

Figure 9: Time and days students spent on learning. (a) Monthly average learning time per day and standard error. (b) Monthly average learning days and standard error.
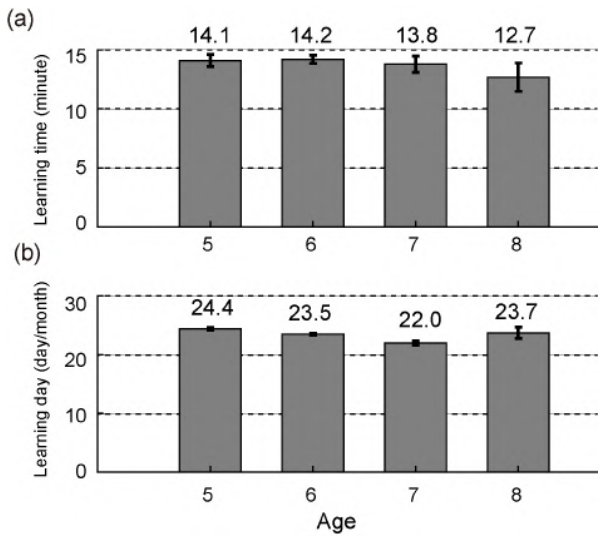

Figure 10: Average learning time and days by age. (a) Average learning time per day and standard error by age. (b) Average learning days per month and standard error by age.

was nearly the same. The students aged five, six, seven, and eight constituted respectively $29 \%, 49 \%, 19 \%$, and $3 \%$ of the students under consideration.

\subsubsection{Analysis Results on Spent Time}

The time spent in a day for learning on the app was derived from the learning history included in the data as follows. SoroTouch transitions to the screen that provides the problems once the user touches on the problem icon. The user must continuously solve a set of math problems corresponding to each specific problem icon. Measurement of the time spent on learning starts from when the problem icon is touched until the completion of the set of problems. For instance, a student taps on the problem icon five times a day, completing five sets of problems. In this case, the learning time measured for the day includes the total time spent attempting five times, each of the times counting from touching the icon until all problems were completed. Incorporation of "Time.realtimeSinceStartup," a feature offered by Unity (Unity Technologies), to SoroTouch programs enables the determination of the time spent between touching the problem icon and completion.

Figure 9 (a) exemplifies the average monthly learning time with the data taken from September 2016 to December 2018. During this period, the shortest recorded was 8.7 minutes per day in September 2016, and the longest was 24.0 minutes per day recorded in November 2017. The average total data taken within this period was 
14.1 minutes per day. Secondly, the average monthly days spent on learning within the period of September 2016 to December 2018 is portrayed in Figure 9 (b). The least number of days spent during this period was 21.2 days per month recorded in August 2017, while the greatest number of days spent was 24.8 days per month recorded in March 2018. The average of all the data taken within this period was 23.5 days per month. Furthermore, Figure 10 depicts the average time and days spent on learning, separated by age. The average time spent on learning per day fell between 12.7 and 14.2 minutes, and the average days with learning per month fell between 22.0 and 24.4 days. Considering other extracurricular activities common to students in this age range, SoroTouch offers a daily habit of gradual learning without excessive workload. These facts inevitably lead to the conclusion that the purpose of daily learning habits is achieved by SoroTouch learners.

\section{CONCLUSIONS}

This paper reports on Digika Co. Ltd.'s implementation of their iPad app, SoroTouch, in the teaching and learning of the abacus and its effectiveness by analyzing quantitative data. We discussed the expected effects of implementing IT systems in education, focusing on the optimization of curriculum and interface, managing individual student learning data history, and standardization of education. The Method section elaborates on the history of the development of SoroTouch and gives an overview of its characteristics, emphasizing on its two key features: "Visible Mode" and "Invisible Mode." Within the SoroTouch curriculum, "Visible Mode" trains accurate hand movements, while "Invisible Mode" helps to teach learn to image the positioning of the beads in the mind, eventually enabling mental calculation. In the Evaluation of SoroTouch section, the research confirms the procedures on short-term acquisition of mental calculation. According to the data, SoroTouch learners, on average, spend 10 to 20 minutes per day and 25 days per month for learning with SoroTouch. Differences were not apparent, as the earlier results show, comparing each student in terms of learning time, day, and age. In other words, the outcome conveys that the learning time does not change due to the differences in individual's natural factors nor their age. Out of the withdrawing students in 2018, numbers revealed that $62 \%$ had attained mental calculation abilities. Moreover, students enrolled after the year of 2014 proved to master mental calculation in less than 20 months on the average.

\section{APPENDIX}

\subsection{Definition of Mastering Mental Calcula- tion}

In this paper, "mastering mental calculation" is defined as either passing the third class Global Mental Calculation Test, organized by Digika Co. Ltd., or completing all 720 missions in the SoroTouch curriculum. To achieve either of them, students is required to acquire the capability to correctly answer more than $80 \%$ of problems that consist of four types of calculations: adding and subtracting 8 twodigit numbers (e.g., $45+97-16+50+38-24+82+78$ ) and 4 three-digit numbers (e.g., $515+366-760+932)$, multiplying threedigit $\times$ one-digit numbers (e.g., $648 \times 8$ ), and dividing four-digit $\div$ one-digit numbers (e.g., $5201 \div 7$ ), using abacus style mental calculation. All the calculations are given in both Listening and Reading style problems and must be solved in the same skilled manner. The time taken on listening problems alters relying upon the speed of dictation 3

\footnotetext{
${ }^{3}$ The dictation speed for Listening problems can be shown in the clip: https://youtu.be/eU7jMC75oY0
}

\subsubsection{Requirements to Complete all 720 Missions}

Students must at least solve the problems listed below. (Addition/Subtraction)

Reading: 8 two-digits 3 questions

Listening: 8 two-digits 6 questions

Reading: 4 three-digits 2 questions

Listening: 4 three-digits 4 questions

* The maximum number of correct answers to subtraction problems is four times out of the 8 two-digits, and two times out of the 4 three-digits

\section{(Multiplication)}

Reading: 3 digits $\times 1$ digit 5 questions

Listening: 3 digits $\times 1$ digit 5 questions

(Division)

Reading: 4 digits $\div 1$ digit 5 questions

Listening: 4 digits $\div 1$ digit 5 questions

The qualification needed to complete the 720 missions is to exceed the necessary percentage of correct answers to the listed questions above 30 times, repetitively. All of the given questions are different, randomly chosen from a group of countless problems prepared beforehand. The sufficient percentage of problems answered correctly is $80 \%$ as of January 15,2018 , and $85 \%$ thereafter.

\subsubsection{Requirements to Pass GMCT 3rd Class}

The test provides the problems given below-each must be answered within five minutes.

(Addition/Subtraction)

Reading: 8 two-digits 5 questions, less than 8 two-digits 55 questions (total 10 questions)

Listening: 8 two-digits 2 questions, 6 two-digits one question, 4 three-digits one question

Reading: 4 three-digits 5 questions

Listening: 4 three-digits one question

* The maximum number of correct answers to subtraction problems is two times out of the 8 two-digits, and one time out of the 4 threedigits.

(Multiplication)

Reading: 3 digits $\times 1$ digit 10 questions

Listening: 3 digits $\times 1$ digit 3 questions

(Division)

Reading: 4 digits $\div 1$ digit 10 questions

Listening: 4 digits $\div 1$ digit 3 questions

In the terms mentioned above, (1) "Percentage of Correct Answers for Addition and Subtraction (Reading)," (2) "Percentage of Correct Answers for Multiplication and Division (Reading)," (3) "Percentage of Correct Answers for Addition, Subtraction, Multiplication, and Division (Listening)," (4) "Percentage of Correct Answers for the whole," the passing rate is $80 \%$ out of all the sections in (1)(4). The time limit for each of the sections (1), (2), and (3) is five minutes. The problems given are varied.

\section{ACKNOWLEDGMENTS}

We wish to thank all students and their parents who support SoroTouch. We thank Miki Murakoshi, Miho Satou, Kaho Tsuchida, Ayumi Nishiyama, Takayo Izumikawa, Takuma Ota, Nami Kimura 
and David Kaschub for their contribution to the development and application of SoroTouch.

\section{REFERENCES}

[1] Lembke E. S. Conoyer S. J., Foegen A. Early numeracy indicators examining predictive utility across years and states. Remedial and Special Education., 37:159-171, 2016.

[2] Geary D. C. Cognitive predictors of achievement growth in mathematics: A 5-year longitudinal study. Developmental Psychology, 47(6):1539-1552, 2011.

[3] Nancy C Jordan, David Kaplan, Maria N Locuniak, and Chaitanya Ramineni. Predicting First-Grade Math Achievement from Developmental Number Sense Trajectories. 22(1):36-46, 2007.

[4] Nancy C. Jordan, Joseph Glutting, and Chaitanya Ramineni. The importance of number sense to mathematics achievement in first and third grades. Learning and Individual Differences, 20(2):82-88, 2010.

[5] Bert De Smedt, Lieven Verschaffel, and Pol Ghesquière. The predictive value of numerical magnitude comparison for individual differences in mathematics achievement. Journal of Experimental Child Psychology, 103(4):469 - 479, 2009. Special Issue: Typical Development of Numerical Cognition.

[6] Eric M. Stickney, Lindsay B. Sharp, and Amanda S. Kenyon. Technology-Enhanced Assessment of Math Fact Automaticity: Patterns of performance for low- and typically achieving students. Assessment for Effective Intervention, 37(2):84--94, 2012.

[7] Takashi Kojima. The Japanese abacus: its use and theory. Charles E. Tuttle Company, Inc., Rutland, Vermont \& Tokyo, Japan, 1954.

[8] Ministry of Education, Culture, Sports, Science and Technology, Japan. Shougakko gakushuu shido youryo, 2019. last accessed September 2019.

[9] David Barner, George Alvarez, Jessica Sullivan, Neon Brooks, Mahesh Srinivasan, and Michael C. Frank. Learning Mathematics in a Visuospatial Format: A Randomized, Controlled Trial of Mental Abacus Instruction. Child Development, 87(4):1146-1158, 2016.

[10] Michael C. Frank and David Barner. Representing exact number visually using mental abacus. Journal of Experimental Psychology: General, 141(1):134-149, 2012.

[11] Takashi Hanakawa, Manabu Honda, Tomohisa Okada, Hidenao Fukuyama, and Hiroshi Shibasaki. Neural correlates underlying mental calculation in abacus experts: A functional magnetic resonance imaging study. NeuroImage, 19(2):296-307, 2003.

[12] Giyoo Hatano and Keiko Osawa. Digit memory of grand experts in abacus-derived mental calculation. Cognition, 15(1):95 - 110, 1983.

[13] Giyoo Hatano, Shizuko Amaiwa, and Keiko Shimizu. Formation of a mental abacus for computation and its use as a memory device for digits: A developmental study. 1987.

[14] Takeshi Hatta and Mayumi Miyazaki. Visual imagery processing in japanese abacus experts. Imagination, Cognition and Personality, 9(2):91-102, 1989.

[15] James W.Stigler. "mental abacus": The effect of abacus training on chinese children's mental calculation. Cognitive Psychology, 16(2):145-176, 1984.

[16] Chika Yamauchi. Program, apparatus and method for support of abacus calculation mental arithmetic learning. Japan Patent JP5893105, March 2016.
[17] Digika Co. Ltd. Sorotouch interface., 2019. https://youtu.be/T1rZVps18t4 last accessed January 2020.

[18] Digika Co. Ltd. Listening practice of sorotouch., 2019. https://youtu.be/VDWaold6JZk last accessed January 2020. 